\title{
CONVERGENCE OF MULTIPLE FOURIER SERIES: MAIN RESULTS AND UNSOLVED PROBLEMS
}

\author{
M. I. DYACHENKO \\ Department of Mathematics and Mechanics, Moscow State University \\ Vorob'evi Gory, Moscow, Russia 119899 \\ E-mail:dyach@mail.ru
}

It is well known that the theory of multiple trigonometric series developed under the strong influence of Zygmund's and Marcinkiewicz's ideas. I'd like to dwell on one of the most interesting problems of this theory which, in my opinion, is convergence of these series. In general I would say that some theorems for multiple Fourier series are easily obtained from the corresponding one-dimensional theorems by induction, or we can establish them by virtually transferring the proofs but slightly complicating them. It stands to reason that theorems of this kind can be of use for constructing the theory but they do not provoke any particular interest. Thus, in considering convergence of multiple Fourier series such theorems make but a small portion. Why it is so will be made clear in what follows.

So, suppose that a function of $m$ variables $(m \geq 2) f(\mathbf{x}) \in L\left(T^{m}\right)(T=[-\pi, \pi))$, $2 \pi$-periodic in each variable, is expanded in a multiple Fourier series

$$
\sum_{\mathbf{n} \in Z^{m}} a_{\mathbf{n}}(f) e^{i \mathbf{n x}},
$$

where $\mathbf{n x}=n_{1} x_{1}+n_{2} x_{2}+\ldots+n_{m} x_{m}$.

If $1 \leq p<\infty$ and $f \in L_{p}\left(T^{m}\right)$ then we define

$$
\|f\|_{p}=\frac{1}{(2 \pi)^{m}}\left(\int_{T^{m}}|f(\mathbf{x})|^{p} d \mathbf{x}\right)^{\frac{1}{p}} .
$$

If $f \in L_{\infty}\left(T^{m}\right) \equiv C\left(T^{m}\right)$, then

$$
\|f\|_{\infty}=\max _{\mathbf{x} \in T^{m}}|f(\mathbf{x})|
$$

2000 Mathematics Subject Classification: Primary 42B08.

The paper is in final form and no version of it will be published elsewhere. 
Further, if $k$ is a natural number, then the $k$-th difference of $f$ at $\mathbf{x}$ with step $\mathbf{t}$ is

$$
\Delta_{k}(f, \mathbf{x}, \mathbf{t})=\sum_{r=0}^{k}(-1)^{r} C_{k}^{r} f(\mathbf{x}+r \mathbf{t})
$$

and the modulus of smoothness of order $k$ in the space $L_{p}\left(T^{m}\right)$ is

$$
\omega_{k}(f, \delta)_{p}=\sup _{\mathbf{t} \in R^{m}:|\mathbf{t}| \leq \delta}\left\|\Delta_{k}(f, \mathbf{x}, \mathbf{t})\right\|_{p} .
$$

Speaking of the convergence of series (1) we should first of all answer two questions.

1. The first question is: in what metric (or in any other sense) the convergence is considered.

2. The second one is: the convergence of what partial sums is meant.

The former question naturally arises in the one-dimensional case, whereas the latter refers entirely to the multidimensional situation. I shall limit myself to examining convergence in the metrics of the $L_{p}\left(T^{m}\right)$-spaces $\left(1 \leq p \leq \infty, L_{\infty} \equiv C\right)$ and convergence almost everywhere. As for the form of partial sums, it is common knowledge that most widely used are the rectangular ones:

$$
S_{\mathbf{N}}(\mathbf{x})=\sum_{\left|k_{1}\right| \leq N_{1}} \cdots \sum_{\left|k_{m}\right| \leq N_{m}} a_{\mathbf{k}} e^{i \mathbf{k x}},
$$

where $\mathbf{N} \in Z^{m} \cap[0,+\infty)^{m}$; (if $\mathbf{N}=(r, \ldots, r)$, then we shall call such sums cubic) and also spherical:

$$
S_{R}(\mathbf{x})=\sum_{|\mathbf{k}| \leq R} a_{\mathbf{k}} e^{i \mathbf{k x}} .
$$

Accordingly, if there exist

$$
\lim _{\min _{1 \leq j \leq m} N_{j} \rightarrow \infty} S_{\mathbf{N}}(\mathbf{x})=\alpha, \quad \lim _{r \rightarrow \infty} S_{(r, \ldots, r)}(\mathbf{x})=\alpha, \quad \lim _{R \rightarrow \infty} S_{R}(\mathbf{x})=\alpha,
$$

then we say that series (1) converges at $\mathbf{x}$ to $\alpha$ over rectangles (in the Pringsheim sense), over cubes and over spheres respectively. We can also give analogous definitions for the convergence in $L_{p}\left(T^{m}\right)$-spaces.

In what follows we shall make it clear that rectangular and spherical partial sums behave quite differently with respect to convergence. Actually there are other ways of defining convergence of multiple series such as convergence over polyhedrons, over hyperbolic crosses, etc. Therefore the definition of convergence including all the above mentioned types seems to be of considerable interest. Note that in all definitions of convergence, the sets over which the sums are taken are symmetric with respect to all coordinate hyperplanes, and they contain, together with every point, an integer parallelepiped whose vertices are at this point and at the origin. Here it seems relevant to give the following definitions.

Definition 1. Let $U \subset Z^{m}$ be a bounded set. Then we say that $U \in A$ if from the fact that $\mathbf{k} \in U$ it follows that

$$
\prod_{j=1}^{m}\left[-\left|k_{j}\right|,\left|k_{j}\right|\right] \cap Z^{m} \subseteq U .
$$


Definition 2. We say that the numerical series

$$
\sum_{\mathbf{n} \in Z^{m}} c_{\mathbf{n}}
$$

$u$-converges to the number $\alpha$ if for any $\varepsilon>0$ there exists a number $M$ such that for every $U \in A$ for which $\left\{\mathbf{n} \in Z^{m}:|\mathbf{n}| \leq M\right\} \subseteq U$, we have

$$
\left|\sum_{\mathbf{n} \in U} c_{\mathbf{n}}-\alpha\right|<\varepsilon
$$

It is evident that if the series is $u$-convergent, then it is convergent in the Pringsheim sense, over spheres, over hyperbolic crosses, etc. The definition of such convergence was originally used by F. G. Arutyunyan [1], [2] when representing functions of several variables by multiple trigonometric series.

As is known, the study of convergence of the trigonometric Fourier series in the $L_{p^{-}}$ metric is simpler than that of convergence almost everywhere. For rectangular sums the results are best possible.

Theorem A (K. Sokół-Sokołowski [3]). If $1<p<\infty$ and $f \in L_{p}\left(T^{m}\right)$, then the Fourier series of $f$ converges to $f$ in the Pringsheim sense in the $L_{p}$-metric.

Theorem B (L. V. Zhizhiashvili [4]). If $f \in C\left(T^{m}\right)\left(f \in L\left(T^{m}\right)\right)$ and

$$
\omega(f ; \delta)_{\infty}=o\left(\left(\ln \frac{1}{\delta}\right)^{-m}\right) \quad\left(\omega(f ; \delta)_{1}=o\left(\left(\ln \frac{1}{\delta}\right)^{-m}\right)\right)
$$

as $\delta \rightarrow+0$, then the Fourier series of $f$ uniformly (in the L-metric) converges to $f$ in the Pringsheim sense. Moreover, if in condition (2) we replace o by $O$, then the assertion is no longer be true even for convergence over cubes.

Similar theorems have been established by L. V. Zhizhiashvili in terms of partial and mixed moduli of continuity.

For the spherical partial sums of the Fourier series of $f \in L_{p}\left(T^{m}\right)$ L. Schwartz ([5], for $\left.p \notin\left(\frac{2 m}{m+1}, \frac{2 m}{m-1}\right)\right)$ and Ch. Fefferman ([6], for $p \neq 2$ ) have established that, generally speaking, they are divergent in this metric. Further, V. A. Ilyin [7] has found that the condition $\omega(f ; \delta)_{\infty}=o\left(\delta^{\frac{m-1}{2}}\right)$ as $\delta \rightarrow+0$ is sufficient for the Fourier series of $f \in C\left(T^{m}\right)$ to uniformly converge to $f$ over spheres. Here the exponent $\frac{m-1}{2}$ cannot be replaced by $\frac{m-1}{2}-\varepsilon$ with $\varepsilon>0$.

In addition, for the two-dimensional case we have V. A. Yudin's result [8] on the norm of the operator of spherical partial sums which implies the following result:

Theorem C (V. A. Yudin). a) If $m=2, p \in\left[\frac{4}{3}, 4\right], p \neq 2, f \in L_{p}\left(T^{2}\right)$ and $\omega(f ; \delta)_{p}=$ $o\left(\left(\ln \frac{1}{\delta}\right)^{-5|1 / / 2-1 / / p|}\right)$ as $\delta \rightarrow+0$, then the Fourier series of $f$ is convergent to $f$ over spheres in the $L_{p}$-metric.

b) If $m=2, p \in\left[1, \frac{4}{3}\right), f \in L_{p}\left(T^{2}\right)$ and $\omega(f ; \delta)_{p}=o\left(\delta^{2 / / p-3 / / 2}\left(\ln \frac{1}{\delta}\right)^{-5(1-1 / / p)}\right)$ as $\delta \rightarrow+0$, then the Fourier series of $f$ is convergent to $f$ over spheres in the $L_{p}$-metric.

c) If $m=2, p \in(4, \infty), f \in L_{p}\left(T^{2}\right)$ and $\omega(f ; \delta)_{p}=o\left(\delta^{1 / / 2-2 / / p}\left(\ln \frac{1}{\delta}\right)^{-5 / / p}\right)$ as $\delta \rightarrow+0$, then the Fourier series of $f$ is convergent to $f$ over spheres in the $L_{p}$-metric. 
The questions whether this theorem is best possible and whether it can be extended to higher dimensional spaces are still open. These problems present considerable interest but they seem to be extremely hard to solve.

It is interesting to compare the theorems by V. A. Ilyin and V. A. Yudin with the following results on $u$-convergence (M. I. Dyachenko [9]). As a preliminary, we shall quote the definition of S. M. Nikolski's classes.

Definition 3. Let $1 \leq p \leq \infty$ and $\alpha>0$. Then we define $H_{p}^{\alpha}\left(T^{m}\right)=\left\{f \in L_{p}\left(T^{m}\right)\right.$ : for all $k>\alpha$ we have $\omega_{k}(f, \delta)_{p}=O\left(\delta^{\alpha}\right)$ when $\left.\delta \rightarrow{ }_{+} 0\right\}$. If here $O$ is changed to $o$ then we denote the corresponding class by $h_{p}^{\alpha}\left(T^{m}\right)$.

Theorem D (M. I. Dyachenko). If $m \geq 2,1 \leq p \leq \infty, p \neq 2$ and the function $f \in h_{p}^{(m-1)|1 / / 2-1 / / p|}\left(T^{m}\right)$, then the Fourier series of $f$ u-converges in $L_{p}\left(T^{m}\right)$-metric.

TheOrem E (M. I. Dyachenko). If $m \geq 2$ and $1 \leq p \leq \infty, p \neq 2$ then there exists a function $f \in H_{p}^{(m-1)|1 / / 2-1 / / p|}\left(T^{m}\right)$ whose Fourier series u-diverges in $L_{p}\left(T^{m}\right)$-metric. Moreover, for $p=\infty$ the Fourier series in the corresponding example u-diverges everywhere.

Hence, in particular, it follows that in the uniform metric, convergence over spheres is one of the worst from the point of view of the width of the class for which it takes place.

Now we proceed to the discussion of convergence almost everywhere. The remarkable results by L. Carleson [10], R. Hunt [11] and P. Sjölin [12] on the a.e. convergence of one-dimensional Fourier series have been generalized to the case of cubic partial sums (N. R. Tevzhadse [13], Ch. Fefferman [14], P. Sjölin [15]). At present the optimal positive result here belongs to N. A. Antonov [16].

Theorem F (N. A. Antonov). If $f \in L\left(\ln ^{+} L\right)^{m} \ln ^{+} \ln ^{+} \ln ^{+} L\left(T^{m}\right)$, then the Fourier series of $f$ a.e. converges to $f$ over cubes.

On the other hand, S. V. Konyagin [17] (1989) has shown that if a function $\varphi(t)$ satisfies some conditions of regularity (for instance, $\varphi(t)$ happens to be the product of powers of iterations of logarithm) and

$$
\int_{2}^{\infty} \frac{\varphi(t)}{t^{2}(\ln t)^{m}} d t<\infty,
$$

then there exists a function $f \in \varphi(L)\left(T^{m}\right)$ whose cubic partial sums of the Fourier series unboundedly diverge almost everywhere.

So the "gap" between positive and negative results here approximately amounts to a logarithm. Until recently this was the situation in the one-dimensional case, too, but recently S. V. Konyagin [18] has obtained the following theorem.

TheOrem G (S. V. Konyagin). For every convex function $\varphi(t)=o\left(t \frac{\sqrt{\ln t}}{\sqrt{\ln \ln t}}\right)$ as $t \rightarrow \infty$ one can find a function $f \in \varphi(L)(T)$ with everywhere diverging trigonometric Fourier series.

The pattern of proving Theorem $\mathrm{G}$ also allows us to establish a similar result in terms of integral moduli of continuity. 
It is most probable that Theorem $\mathrm{G}$ can be modified for the multidimensional case, too, but all the same a "gap" between positive and negative results remains, and the problem of its elimination seems to be the greatest challenge.

Concerning the convergence in the sense of Pringsheim, in the first place we should mention the result of Ch. Fefferman [19] who established that there exists a continuous function of two variables whose Fourier series diverges everywhere in Pringsheim sense. Later M. Bahbuh and E. M. Nikishin [20] specified the statement by establishing that if we limit ourselves to considering divergence over a set of positive measure, then the modulus of continuity of such a function can be of the order of $O\left((\ln 1 / / \delta)^{-1}\right)$ as $\delta \rightarrow+0$. These conclusions were continued by the following results.

Theorem H (L. V. Zhizhiashvili [21]). If $f \in L_{2}\left(T^{m}\right)$ and for some $\varepsilon>0$

$$
\omega(f ; \delta)_{2}=O\left(\left(\ln \frac{1}{\delta}\right)^{-m / / 2-\varepsilon}\right)
$$

as $\delta \rightarrow+0$, then the Fourier series of $f$ converges almost everywhere to $f$ in the Pringsheim sense.

Theorem I (A. N. Bakhvalov [22]). If $m=2 k, k \in \mathbf{N}$, then there exists a function $f \in C\left(T^{m}\right)$ with $\omega(f ; \delta)_{\infty}=O\left(\left(\ln \frac{1}{\delta}\right)^{-m / / 2}\right)$ as $\delta \rightarrow_{+} 0$ whose Fourier series everywhere diverges in the Pringsheim sense.

Hence we conclude that in the spaces of even dimension the "gap" between positive and negative results in the logarithmic scale is nonexistent, while in the spaces of odd dimension $\geq 3$ it is equal to $\sqrt{\ln \frac{1}{\delta}}$. Here naturally arises the problem of improving the available results (though this problem for the spaces of even dimension has not yet been completely solved). Also the following K. I. Oskolkov two-dimensional result [23] so far has had no multidimensional analogues.

Theorem J (K. I. Oskolkov). If $f \in C\left(T^{2}\right)$ and $\omega(f ; \delta)_{\infty}=o\left(\left(\ln \frac{1}{\delta}\right)^{-1}\left(\ln \ln \ln \frac{1}{\delta}\right)^{-1}\right)$ as $\delta \rightarrow+0$, then the Fourier series of $f$ converges a.e. in the Pringsheim sense.

The study of these problems is closely connected with that of Weyl multipliers for convergence almost everywhere. I cannot discuss it here in greater detail. I can only say that at present the only final result available is for $m=2$ (P. Sjölin [15], E. M. Nikishin [24]). It boils down to the following: the sequence $\left\{\ln ^{2}\left(\min \left(\left|n_{1}\right|,\left|n_{2}\right|\right)+2\right)\right\}_{n_{1}, n_{2} \in Z}$ is a sharp Weyl multiplier for the Pringsheim convergence almost everywhere.

Of great interest are also the results on sufficient smoothness conditions for almost everywhere Pringsheim convergence of Fourier series of integrable functions.

Theorem K (L. V. Zhizhiashvili [21], A. M. Stokolos [25]). If $f \in L\left(T^{m}\right)$ and

$$
\int_{0}^{1} \frac{\omega(f ; t){ }_{1}\left(\ln \frac{1}{t}\right)^{m-1}}{t} d t<\infty,
$$

then the Fourier series of $f$ converges almost everywhere in the Pringsheim sense. In particular, it is so if $\omega(f ; \delta)_{1}=O\left(\left(\ln \frac{1}{\delta}\right)^{-m-\varepsilon}\right)$ as $\delta \rightarrow_{+} 0$, where $\varepsilon>0$. 
Theorem L (S. V. Konyagin [26]). If

$$
\omega(t)\left(\ln \frac{1}{t}\right)^{m-1} \ln \ln \frac{1}{t} \rightarrow \infty
$$

as $t \rightarrow+0$, then there exists a function $f \in H_{1}^{\omega}\left(T^{m}\right)$ whose Fourier series cubically diverges almost everywhere.

The final theorems here as well as in the one-dimensional case are as yet unknown. Moreover, of interest is the question if it is possible, in terms of moduli of continuity in $L$, to tell the difference between the behavior of rectangular and cubic partial sums of Fourier series. Then it is interesting to establish the specific conditions for almost everywhere Pringsheim convergence in $L_{p}$-spaces, $p \neq 1,2$.

One of the unsolved major problems of multidimensional harmonic analysis is this: must the Fourier series of a function $f \in L_{2}\left(T^{m}\right), m \geq 2$ spherically converge almost everywhere? Here the best result yet known belongs to B. I. Golubov [27].

Theorem M (B. I. Golubov). If $f \in L_{2}\left(T^{m}\right)$ and for some $\varepsilon>0, \omega(f ; \delta)_{2}=$ $O\left(\left(\ln \frac{1}{\delta}\right)^{-1-\varepsilon}\right)$ as $\delta \rightarrow+0$, then the Fourier series of $f$ spherically converges to $f$ a.e.

Similar results in $L_{p}$-spaces, $p \neq 2$ are so far unknown.

Sufficient smoothness conditions of $u$-convergence a.e., sharp in the power scale, have been established by M. Dyachenko [28] in $L\left(T^{2}\right)$ and $L_{2}\left(T^{2}\right)$-spaces. Multidimensional analogues are yet unknown. Here I formulate only one of these statements.

Theorem N (M. I. Dyachenko). If $f \in L_{2}\left(T^{2}\right)$ and

$$
\int_{0}^{1} \frac{\left(\omega(f, t)_{2}\right)^{2}}{t^{2}} d t<\infty,
$$

then the Fourier series of $f$-converges almost everywhere.

That this statement is best possible in the power scale follows from Theorem E.

\section{References}

[1] F. G. Arutunian, Representation of functions by multiple series, Reports of Armenian Acad. Sci. 64 (1977), 72-76 (in Russian).

[2] F. G. Arutunian, Representation of measurable functions of many variables by multiple Fourier series, Matem. Sbornik 126 (1985), 267-285 (in Russian); English transl.: Math. USSR-Sb. 54 (1986), 259-277.

[3] K. Sok/o/l-Soko/lowski, On trigonometric series conjugate to Fourier series of two variables, Fund. Math. 34 (1947), 166-182.

[4] L. V. Zhizhiashvili, On some questions of the theory of simple and multiply trigonometric and orthogonal series, Usp. Matem. Nauk 28 (1973), no. 2, 65-119 (in Russian); English transl.: Russian Math. Surveys 28 (1973), no. 2, 65-127.

[5] L. Schwartz, Sur les multiplicateurs de $F L^{p}$, Kungl. Eysiograf. Sallsikapets Lund Fohandlingar. 22 (1953), 692-695.

[6] Ch. Fefferman, The multiplier problem for the ball, Ann. Math. 94 (1971), 330-336. 
[7] V. A. Ilyin, On the convergence of the expansions with respect to the eigenfunctions of the Laplace operator, Usp. Matem. Nauk 13 (1958), no. 1, 87-180 (in Russian).

[8] V. A. Yudin, Spherical sums of the Fourier series in $L_{p}$, Mat. Zametki 46 (1989), 145-152 (in Russian); English transl.: Math. Notes 46 (1989), 675-680.

[9] M. I. Dyachenko, u-convergence of multiple Fourier series, Izv. RAN. Ser. Matem. 59 (1995), 128-142 (in Russian); English transl.: Izvestiya: Math. 59 (1995), 353-366.

[10] L. Carleson, On convergence and growth of partial sums of Fourier series, Acta Math. 116 (1966), 135-157.

[11] R. A. Hunt, On the convergence of Fourier series, in: Orthogonal Expansions and Their Continuous Analogues (Edwardsville, Ill., 1967), Southern Ill. Univ. Press, Carbondale, Ill., 1968, 233-255.

[12] P. Sjölin, An inequality of Paley and convergence almost everywhere of Walsh-Fourier series, Ark. Mat. 7 (1969), 551-570.

[13] N. R. Tevzadze, On the convergence of the double Fourier series of a square summable function, Soobsh. AN GSSR 58 (1970), 277-279 (in Russian).

[14] Ch. Fefferman, On the convergence of multiple Fourier series, Bull. Amer. Math. Soc. 71 (1971), 744-745.

[15] P. Sjölin, Convergence almost everywhere of certain singular integrals and multiple Fourier series, Ark. Mat. 9 (1971), 65-90.

[16] N. Yu. Antonov, Convergence of Fourier series, East J. Approxim. 2 (1996), 187-196.

[17] S. V. Konyagin, On the divergence of the subsequence of partial sums of the multiple trigonometric Fourier series, Trudy MIAN 190 (1989), 102-116 (in Russian); English transl.: Proc. Steklov Inst. Mat. 190 (1992), 107-122.

[18] S. V. Konyagin, On everywhere divergence of trigonometric Fourier series, Matem. Sbornik 191 (2000), 103-126 (in Russian); English transl.: Sbornik: Math. 191 (2000), 97-120.

[19] Ch. Fefferman, On the divergence of multiple Fourier series, Bull. Amer. Math. Soc. 71 (1971), 191-195.

[20] M. Bahbuh and E. M. Nikishin, The convergence of double Fourier series of continuous functions, Sibirsk. Mat. Zh. 14 (1973), 1189-1199 (in Russian); English transl.: Siberian Math. J. 14 (1973), 832-839 (1974).

[21] L. V. Zhizhiashvili, On the convergence of multiple trigonometric Fourier series, Soobsh. AN GSSR 80 (1975), 17-19 (in Russian).

[22] A. N. Bakhvalov, On the divergence everywhere of the Fourier series of continuous functions of several variables, Matem. Sbornik 188 (1997), 45-62 (in Russian); English transl.: Sbornik: Math. 188 (1997), 1153-1170.

[23] K. I. Oskolkov, Estimation of the rate of approximation of a continuous function and its conjugate by Fourier sums on a set of full measure, Izv. AN SSSR 38 (1974), 1393-1407 (in Russian).

[24] E. M. Nikishin, Weil multipliers for multiple Fourier series, Matem. Sbornik 89 (1972), 340-348 (in Russian); English transl.: Math. USSR-Sb. 18 (1972), 351-360.

[25] A. M. Stokolos, On the strong differentiation of the integrals of functions from Hölder classes, Matem. Zametki 55 (1994), 84-104 (in Russian); English transl.: Math. Notes 55 (1994), 57-70.

[26] S. V. Konyagin, On divergence of trigonometric Fourier series over cubes, Acta Sci. Math. Hung. 61 (1995), 305-329. 
[27] B. I. Golubov, On the convergence of spherical Riesz means of the multiple Fourier series, Matem. Sbornik 96 (1975), 1254-1278 (in Russian); English transl.: Math. USSR-Sb. 25 (1975), 177-197 (1976).

[28] M. I. Dyachenko, U-convergence almost everywhere of double Fourier series, Matem. Sbornik 186 (1995), 47-64 (in Russian); English transl.: Sbornik: Math. 186 (1995), 47-64. 